\title{
The Effect of Sleep Duration on Short-term Memory
}

\author{
Rui $\mathrm{Xu}^{1, \mathrm{a},{ }^{*}, \dagger}$ Yang $\mathrm{Yi}^{2, \mathrm{~b}, *},{ }^{*}$ Xinlan Zhang ${ }^{3, \mathrm{c}, *, \dagger}$ \\ ${ }^{1}$ College of Pharmacy, East China University of Science and Technology, Shanghai, China \\ ${ }^{2}$ College of Art and Science, University of Washington, Seattle, Washington, United States of America \\ ${ }^{3}$ College of Liberal Arts, Pennsylvania State University, State College, Pennsylvania, United States of America \\ *Corresponding author.Email: ${ }^{a} 1827979187 @ q q . c o m,{ }^{b y y 0328 @ u w . e d u, ~}{ }^{c x j z 5161 @ p s u . e d u}$
}

${ }^{t}$ These authors contributed equally.

\begin{abstract}
Sleeping time is usually associated with short-term memory, while the mechanisms under this relationship are less understood. This study examined the role of sleeping time in this relationship with cross-sectional data. 24 Chinese between 18 and 30 years old were recruited. Their sleeping time was recorded, and PsychoPy collected the data of memory test. The results showed that (1) the Sleeping time is positively correlated with sleep duration, (2) participants were more likely to remember the words related to emotions. These findings suggest that proper sleeping time can help improve the efficiency of productivity.
\end{abstract}

Keywords: Short-term memory, Sleeping time, Hippocampus, Recognition test.

\section{INTRODUCTION}

Since ancient times, countless philosophers and writers have praised the importance of memory. Danish poet and author Tove Ditlevsen wrote in her novel "Early Spring" (1985), "Memory, that library of the soul from which I will draw knowledge and experience for the rest of my life" [1]. Ditlevsen compares memory to a library of her soul. It can be seen that memory is a unique and valuable asset that is remarkable for every human being, which contains all life values.

\subsection{Importance and classification of memory}

Memory is the ability of the brain to retain and retrieve information based on past experience. It is the fundamental and inherent technology that the brain constructed for human beings to communicate with the world, which helps humans transform chaotic content into orderly knowledge and information through processing.

\subsubsection{Importance of memory}

Memory has a vital role in the survival and development of individuals. Memory maintains human cognition of the internal personality and external understanding of the world. In the process of individual development, memory has a multifaceted function in accumulating experience, repetitive application of knowledge, reasoning, judgment, and adaptation.

Loss of memory function indicates the loss of personal identification and thus the ability to think and learn. The most common case, in reality, is Alzheimer's disease. This neurodegenerative disorder will gradually lose the synapses and neurons in the cerebral cortex, leading to short-term memory loss and various brain dysfunctions, which will eventually cause incapacity or even death.

\subsubsection{Classification of memory}

According to the difference in the retention time of information stored in the brain, Atkinson and Shiffrin used the "Atkinson-Shiffrin memory model" (1968) to classify memory into (1) sensory memory, (2) short-term memory, and (3) long-term memory [2]. The main difference among these three memory systems is the storage time and capacity of information, but they are related to each other in the process of memory processing.

Sensory memory is a certain amount of perceptual information about the outside world when the stimulus occurs, including visual and auditory stimuli. The main feature of sensory memory is that the storage time is very short, which remains for only about $0.25-2$ seconds, and it has a limited storage capacity. Whereas long-term memory refers to the information that is ultimately 
preserved by the brain after multiple processing and screening. It has unlimited storage capacity, so it is also interpreted as a "library" effect on memory.

\subsection{Short-Term Memory}

Short-term memory is a small amount of holding information, which oversees extracting information from the brain without manipulation. It plays the most important functions of memory maintaining, reviewing, and repetition. Short-term memory is responsible to the brain in the information processing system, so it has more storage time and capacity, which can last about 5 seconds to 2 minutes.

Among these three kinds of memories, short-term memory has the most intuitive impact on human beings. In the article, "The Mind and Brain of Short-Term Memory", John Jonides and others (2007) analyzed short-term memory from the perspective of neuroscience. Through (1) the expression of short-term memory and methods to distinguish it from other memories, (2) encoding, maintenance, and retrieval process of shortterm memory, and (3) loss, consolidation model, and interference factors of short-term memory, researchers suggested that short-term memory has a "biological mechanisms that might support psychological processes on a moment-by-moment basis as an item is encoded, maintained over a delay with some forgetting, and ultimately retrieved" [3]. Therefore, short-term memory has a function in the storage and processing of human memory and enhances the ability to understand, accept, and express information in life.

In this study, a memory recognition test will be used to measure participants' short-term memory on vocabulary recognition ability. In addition, vocabularies will be divided into four categories with different implications of meaning, which contains the fields of mood, food, academic major, and preposition. In this way, the experiment could further determine the ability of short-term memory to recognize words with different meanings.

Thus, the present study aims to investigate the factors affecting short-term memory.

\subsection{The Influential Factors of Short-Term Memory}

Many factors affect short-term memory, such as the excitability of the cerebral cortex, level of information processing by the brain, total amount of synapses and neurons in the cortex, and so on. In response to discovering short-term memory performance after experiencing the different amounts of sleep, the factor of sleep duration at night will be induced to this experiment.

\subsection{Sleep Duration}

Sleep duration usually refers to the total amount of sleep a person gets every 24 hours. It can also be interpreted as the amount of rest which the mind and body received in a certain period. Sleep duration is usually measured by a questionnaire or brain wave detection.

In 2014, the Centers for Disease Control and Prevention stipulated that "adults need 7 or more hours of sleep per night for the best health and wellbeing. Short sleep duration is defined as less than 7 hours of sleep per 24-hour period" [4]. It indicates that if an adult wants to receive good mental status and vitality after sleep, one must sleep seven hours or longer. If the sleep duration is less than 7 hours, they can not meet the "healthy sleep" standard defined by CDC. Under the same conditions, teenagers and the elderly need longer sleep duration to meet the health requirements. According to CDC sleep regulation, combining the influences of Chinese culture and the needs of research purposes, the study will investigate sleep duration -respectively through 3 controlled groups with different amounts of sleep duration: 3-6 hours, 6-9 hours, and 9-12 hours.

The amount of sleep duration's impact on humans is reflected in many aspects such as immunity, mental status, and memory capacity. But the most important thing is that the reduction of sleep duration will be accompanied by a significant decrease in the performance of short-term memory-related activities, and these activities are usually correlated with learning and extracting information.

\subsection{The Effects of Sleep Duration on Short- Term Memory}

Short-term memory has many concretization forms of expression in human life, including study, work, etc. A greater amount of sleep duration has a certain effect on the manifestations of short-term memory in life. In a recent study, "Sleep quality, duration, and consistency are associated with better academic performance in college students" performed by Kana Okano and others (2019), researchers experimented on 100 college students with a range of sleep duration from 5 to 9 hours and studied the effect of sleep time on their scores of chemistry test [5]. The data showed a positive linear relationship between sleep time and college students' chemistry test scores, with a relatively large variance (about 25\%) between sleep duration and college students' chemical test scores. The study indicates sleep duration is highly correlated with scholar performance, and college students will achieve higher academic performance with the growth of sleep duration.

The impact of sleep duration on short-term memory is mainly reflected in the capacity, processing performance due to brain activation, and related 
memories' working speed. In previous research, "Functional neuroimaging and behavioral correlations of capacity decline in visual short-term memory after sleep deprivation" (2007), Michael W. L. Chee and Y. M. Lisa Chuah verified the damage of sleep deprivation on shortterm memory from the perspective of neuroimaging. The researchers tested the visual memory of 30 participants after normal sleep and deprivation sleep by visual shortterm memory (VSTM) tasks [6]. Michael and others defined sleep deprivation as a status of sleep duration which exposures to 24 hours without rest. The researchers measured differences in the score of participants on VSTM and visual attention tasks as an expression of short-term memory for the experiment after exposure to sleep deprivation. At the same time, they used the fMRI neuroimaging method to determine the activated area of the brain when it is associated with the dependent variable through the concentrated area of blood flow in the brain. In this way, researchers can accurately measure the activation level within short-term memory-related brain structures. Their findings indicate that the preservation of precuneus load and temporary junction deactivation will appear and increase after sleep deprivation, accompanied by defects in visual processing and visual attention functions, which leads to the loss of short-term memory capacity. Experimental results showed that after 24 hours of sleep deprivation, the activation of precuneus, posterior cingulate cortex, and temporoparietal junction bilaterally decreased. It indicates that after exposure to sleep deprivation, the blood flow speed and neuron responses in the brain tissues associated with short-term memory are reduced, and the brain's ability and capacity to process visual short-term memory are temporarily impaired. On the contrary, activation lost will be reactivated after proper sleep, thereby increasing the capacity and performance of short-term memory. At the same time, the impact of sleep duration is also reflected in the ability of short-term memory to process different types of tasks. For example, when participants perform a memory recognition task, short-term memory accuracy for words with different meanings will be different.

With the same principle, an improvement in sleep duration also can effectively improve the mental state and performance at work for employees. In the context of China, there is a social phenomenon of company system which limits people to reach the healthy sleep duration stipulated by the CDC, which is called the "996 working hour system". It indicates that an employee must be presented at the company to work before 9 am and must leave work after $9 \mathrm{pm}$, even sometimes people are allowed to leave until they finish their job for the day. This social phenomenon has to squeeze the value of employees by reducing sleep and rest time in the work field, thereby improving work efficiency. In such a social environment, even people may receive some monetary compensation, many employees still complain about insufficient sleep time and prolonged fatigue. Michael and others have suggested that "decline in working memory after SD may be strongly influenced by degraded attention" [6]. Michael's finding could also explain these frustrations in job performance that sleep deprivation will significantly reduce short-term memoryrelated brain area and visual processing system activation. Therefore employees will suffer from a depress in the ability to handle their works.

\subsection{The Present Study}

In summary, this study will explore the impact of sleep duration on short-term memory and propose the following hypotheses:

(1) Short-term memory ability will vary according to different vocabulary meanings.

(2) There is a correlation between sleep duration and the manifestations of short-term memory.

As sleep duration increases, participants will likely perform better in short-term memory tests or vice versa.

\section{METHOD}

Participants were asked to use and record their sleeping duration and finish the memory test via PsychoPy right after waking up and reciting all the words.

\subsection{Participant}

The participants included 24 Chinese between 18 and 30 years old $(M=22, S D=3.2)$. They met the criteria for maintaining an adequate level of English. All participants voluntarily participated in this experiment and were randomly assigned to each of the conditions. The sample consisted of 8 males and 16 females. For the academic background, most participants, around $40 \%$ of the participants were high school students, 35\% were undergraduate students, and $25 \%$ were graduate students. Participation was voluntary.

\subsection{Design}

This study was an experimental design that contained one independent variable with three levels, indicating the distinguished sleeping interval as 3-6 hours, 6-9 hours, 912 hours. The dependent variable was short-term memories for listed words in a relatively short time.

\subsection{Materials and apparatus}

\subsubsection{Sleep Duration}

Using sleep duration as an independent variable, the subjects are divided into three groups based on sleep duration, including group 1 sleeping 0-3 hours, group 2 sleeping 3-6 hours, and group 3 sleeping 6-9 hours. 


\subsubsection{Accuracy}

According to the data collected via PsychoPy, the accuracy rate of the test subjects in different periods is counted as the dependent variable.

\subsection{Procedure}

Before the main experiment begins, one member from group three in the cognitive science program performed the test trial to examine the instructions are well-constructed, and the questions are comprehensive. 24 participants were given advance notice about recording their sleeping duration for the previous night. As the participants wake up, they are involved in the experiment by running the memory recognition test in the PsychoPy. The informed consent about the instructions and one's data will be recorded notified in the beginning. In the front, participants selected their corresponding sleeping hours among 5 options of interval for sleeping. The researchers displayed 20 words in random order one at a time; participants need to try processing the information to store it in mind for 2.5 seconds. The following memory test was ready for the participants to select whether they are familiar with the word or not. The tested word was in the middle of the screen; the 2 options, "old" and "new," with directional arrows, are on the 2 sides under the term. False feedbacks will not be presented on the screen but to be included in the data analysis. After the main task, there was a review session for the participants to fill in the survey about their personal information, test performance, sleeping hours, and feedback about the experiment.

\section{RESULTS}

\subsection{Descriptive Statistics}

Table 1. Means, standard deviations of study variables

\begin{tabular}{lcccccc}
\hline & \multicolumn{2}{c}{ Low } & \multicolumn{2}{c}{ Middle } & \multicolumn{2}{c}{ High } \\
\cline { 2 - 7 } & M & SD & M & SD & M & SD \\
& & & & & & \\
\hline Over-all & 0.55 & 0.02 & 0.70 & 0.03 & 0.86 & 0.04 \\
dependent & & & & & & \\
variable & & & & & & \\
Mood & 0.94 & 0.01 & 0.95 & 0.10 & 0.96 & 0.01 \\
Food & 0.91 & 0.01 & 0.91 & 0.01 & 0.92 & 0.01 \\
Major & 0.78 & 0.04 & 0.84 & 0.04 & 0.87 & 0.05 \\
Preposition & 0.84 & 0.01 & 0.85 & 0.01 & 0.86 & 0.01
\end{tabular}

The distribution of the calculated mean shown in Table 1 suggests the previous findings of which accuracy on the memory recognition task will increase as sleeping duration goes up. And the standard deviation of the mean and standard error indicates a relatively small spread out from the mean. If categorizing the memorized words according to their types of meaning, it is obvious that the participants are most likely to remember words related to mood, up to $95 \%$ accuracy. Besides, the accuracy for Food and Preposition were $91.7 \%$ and $85 \%$, respectively. The memory performance of Major was the worst, with only $83 \%$ correctness. These data might suggest that participants may have associative memories of words with specific meanings in the process of memorizing words. This method will effectively improve the quality of memory.

\subsection{The Relationship Between Sleep Duration and Accuracy}

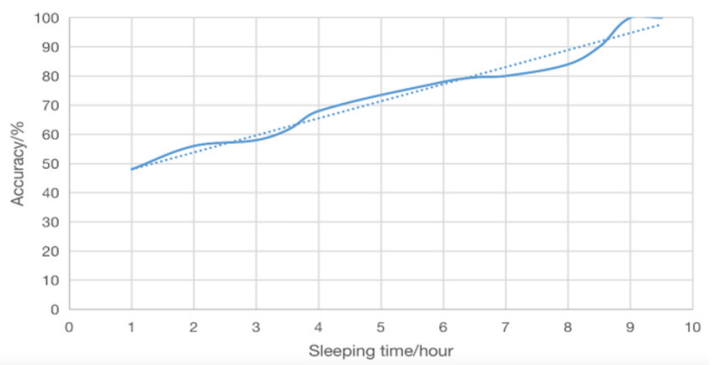

Figure 1. The relationship between sleep duration and accuracy

Figure 1 provides a visualization of a linear function with a slope of positive 4.96 and y-intercept at 42 (The formula is $y=4.96 x+42$ ). The slope of 4.96 represents that the dependent variable, which is accuracy on memory recognition task, will be increased by $4.96 \%$ as sleeping duration at night increase by 1 hour. A directional conclusion can be determined by the distribution and expression of the line of best fit. The longer time participant sleep at night is correlated to better ability of performance on vocabulary recall, and that under the extreme condition without sleep at night all. People could theoretically achieve $42 \%$ accuracy on the memory recognition task.

\section{DISCUSSION}

This study explored the impacts of sleeping time on short-term memory. The inadequacy of sleep time might lead to mental fatigue, impaired decision-making, migraine, and even epilepsy due to the limited time to activate the process in the glymphatic system [7]. The general hypothesis is supported by the data from the result section, which suggested participants were able to recall the significantly increased number of words after they sleep more than 6 hours compared with those whose sleeping time in under 6 hours. The findings were consistent with the previous research that sleeping plays an essential role in declarative memory consolidation [8]. 
The participates' recall performance tends to increase after an interval of sleep.

\subsection{The difference of the task performance on the three sleeping interval}

The research concentrated on three sleeping hours from 0 to 9 hours which sleeping durations were equally distributed into three sections on the performance of the memory recognition test. All the precision rates of recalled words are recorded from the low, middle, and high levels within the assigned sleeping durations. The data table from the result section demonstrated each category for the veracity words under the overall experiment performance, which serves as a function to detect whether the types might influence the results of the memory recognition test.

\subsubsection{Overall performance on the number of recalling words}

In general, the accuracy of recalling words tends to fortify with the increase of each sleeping durations, which demonstrates that the outstanding performance for the short memory is within the 6-9 interval; participants tend to perform neither better nor worse within the 3-6 intervals, whereas the 0-3 break includes the worst accuracy of recalling words. Recent research has been found that the restriction of sleeping time negatively affects adolescents' ability to learn declarative information [9]. In this experiment, the two sets of the group are the 9 hours as the control group in bed compared with 5 hours as the restricted group. The result suggested that memory was significantly worse in the sleep-restricted group. The findings of the present studies are consistent with the previous research that sleeping deprivation might produce misleading information, which influences the accuracy of memory.

\subsubsection{The effect for categories of words on short-term memory}

The data table displayed the significantly growing tendency among participates in memorizing more words related to emotions and food compared with the proposition and majors in the memory recognition test. The previous research has linked to this finding by suggesting that memory benefits for emotional words compared with neutral ones [10]. This general enhancement effect was relatively consistent, occurring in 20 of 24 participates. Besides, recalling the food items is relatively high because of the notion of a real object with the sensory association. Psychologist Hoogeveen and his team found that the neuronal mechanisms are previous underlying observations that sensory associations are essential features of food memory in 2016 [11]. On the contrary, the number of words for both prepositions and majors categorize that participants recall relatively less due to the high similarity between learning and tested words. The categories of the words could play an essential role in the memory recalling test based on the findings above. This discrepancy is likely because some stimulus features would be processed automatically, whereas other features would require effortful processes [12].

\subsection{The influence of sleeping time on short- term memory}

Sleep is related to diverse categories of learning and memory with both explicit and implicit [13]. The human brain has already extensively processed recent memories during the first hours of post-training wakefulness [14]. In the memory recognition task, the participants performed the test on PsychoPy after they were awake for the training to remember the 20 words, which activates the tendency to aggrandize the neural activities in managing the incoming phrase information. The results in figure 1 showed a positive correlation between sleep duration and vocabulary recall accuracy. The importance of adequate sleep in optimal cognitive functioning; sleepdeprived individuals whose sleeping hours are under 6 hours were more likely to incorporate misleading information into their responses during memory retrieval [15]. In the present work, the accuracy of recalling words is relatively lower under 6 hours which corresponded with the previous study. Additionally, inaccurate perception of indicating the no-studied items was weakened after sleep. Compared to participates whose sleeping interval is within 3-6 hours, the group of participates who sleep between 6 to 9 hours demonstrated significantly increased performance with the longer sleeping intervals. The poor performance on recognition memory test within 0-3 hours could be explained by previous research indicating that inadequate sleep tends to lower cognitive manipulation in both the frontal and parietal regions and decrease the connection with the extrastriate visual cortex cause attentional impairments [16]. The participates' attention was not entirely devoted to the learning stage, which causes the poor memory test performance due to the lack of processing incoming information. The accuracy of recalling words was under 50 percent when participates' sleep was deprived. The result is consistent with the previous research that 44 students reduced their academic performance on cognitive tasks due to sleeping deprivation [17]. The participates could be negatively impacted on the memory test because of the irregular sleeping schedule.

\subsection{Limitation and Future Research direction}

This study has potential limitations due to the measurement validity. Firstly, the gender imbalance of the overall participates shifts to $66.6 \%$ of females. The previous research about gender differences in memory recall suggests that females outperform men in memory 
recall overall and for all types of items except masculine ones regarding gender-stereotyped items [18]. Future research should include relatively equal numbers of participants from each gender to demonstrate a more evident difference in experiment performance between females and males; ensure overall accuracy results for the memory recognition task. Second, the sample size is limited to 24 participants. Ideally, three sleeping intervals should have included at least 35 participants based on quantitative research requirements. The minimized sample size could potentially have negatively affected the internal validity of the experiment. Third, no manipulation of the sleeping quality appeared in this current research, which might interfere with the experiment as a confounding variable. The study had no access to neuroimaging techniques due to the limitation of the technology. In future research, the monitor application for sleeping quality on electronic devices could be applied as part of the procedure. Finally, the research concentration is on how the sleeping time would affect short-term memory; the research could be extended to other types of memories as the long-term memory.

According to the standard two-stage memory model that David Marr proposed in 1971, the new information is encoded to a temporary store at the hippocampus before gradually transferring into long-term storage at the neocortex [19]. During the sleeping stage, the participates are not exposed to new information, which activates the connection between cortical neurons, mainly in the hippocampus and the cortical network. The result suggests that increased sleeping hours allow the hippocampus more time to ultimately convert the previous day's short-term memory into long-term memory stored in the neocortex and possibly save storage space in the hippocampus to encode new following information. The current study findings with the previous research are concerning the potential application of memory outside the laboratory. A trendy phenomenon called "996" in the Chinese working environment requires mostly the employees in the IT industry to work for 10 hours from 9 a.m. to 9 p.m. with the six workdays system. "996" does not only against the labor law but also threatens the health of the employees. This work culture prevents the employees from maintaining enough sleeping hours, which might reduce work efficiency due to the limited time for the brain to process the gained information into long-term memory. The working schedule adjustment should be reassigned to obtain adequate sleep at night to complete more professional the distributed task. Given the importance of short-term memory on academic and job performance, a fuller understanding of the impact of sleep on memory consolidation is needed. Other studies are needed to investigate the specific influences of rest on other types of memory, such as episodic, procedural, and working. Furthermore, future studies could extend the possibilities of exploring the role of different stages of sleep such as awake, light and deep on the memory performance with the utilize of neuroimaging techniques displaying the physiological brain functioning and neuronal activities.

\section{CONCLUSION}

Sleeping duration is found to be related to memory. Besides, the accuracy of recalling words under different categories varies a lot. Evidence from this study has revealed that short-time memory is positively correlated with sleep duration. Short-time memory improves with the increase of sleeping duration. Moreover, an obviously growing tendency among participates in memorizing more words related to emotions has occurred in the memory recognition test. In addition, this evidence has significant implications for the workers or the students to which this study paid attention. In other words, more sleeping duration helps improve work efficiency. Thus, adequate sleep can significantly improve the productivity of the whole society.

\section{REFERENCES}

[1] Ditlevsen, Tove. (1986). Early spring. London: The Women's Press

[2] Atkinson, R.C.; Shiffrin, R.M. (1968). "Chapter: Human memory: A proposed system and its control processes". In Spence, K.W.; Spence, J.T. (eds.). The psychology of learning and motivation. 2. New York: Academic Press. pp. 89195.

[3] Jonides, J., Lewis, R. L., Nee, D. E., Lustig, C. A., Berman, M. G., \& Moore, K. S. (2008). The mind and brain of short-term memory. Annual review of psychology, 59, $193-224$ https://doi.org/10.1146/annurev.psych.59.103006.0 93615

[4] Centers for Disease Control and Prevention. (2017, May 2). CDC - Data and Statistics - Sleep and Sleep Disorders. Centers for Disease Control and Prevention.

https://www.cdc.gov/sleep/data_statistics.html.

[5] Okano, K., Kaczmarzyk, J.R., Dave, N. et al. Sleep quality, duration, and consistency are associated with better academic performance in college students. npj Sci. Learn. 4, $16 \quad$ (2019). https://doi.org/10.1038/s41539-019-0055-z

[6] Chee, M., \& Chuah, Y. (2007). Functional Neuroimaging and Behavioral Correlates of Capacity Decline in Visual Short-Term Memory after Sleep Deprivation. Proceedings of the National Academy of Sciences of the United States of America, 104(22), 9487-9492. Retrieved July 8, 2021, from http://www.jstor.org/stable/25427880 
[7] Eugene, A. R., \& Masiak, J. (2015). The. Neuroprotective Aspects of Sleep. MEDtube science, 3(1), 35-40.

[8] J.P. Queille, Backhaus, J., Hoeckesfeld, R., Born, J., Hohagen, F., \& Junghanns, K. (2008). Immediate as well as delayed post learning sleep but not wakefulness enhances declarative memory consolidation in children. Neurobiology of Learning and Memory, 89(1), 76-80. https://doi.org/10.1016/j.nlm.2007.08.010

[9] Cousins, J. N., Sasmita, K., \& Chee, M. (2018). Memory encoding is impaired after multiple nights of partial sleep restriction. Journal of sleep research, 27(1), 138-145. https://doi.org/10.1111/jsr.12578

[10] Kensinger, E.A., Corkin, S. Memory enhancement for emotional words: Are emotional words more vividly remembered than neutral words?. Memory \& Cognition 31, 1169-1180 (2003). https://doi.org/10.3758/BF03195800

[11]Hoogeveen, H. R., Jolij, J., Ter Horst, G. J., \& Lorist, M. M. (2016). Brain Potentials Highlight Stronger Implicit Food Memory for Taste than Health and Context Associations. PloS one, 11(5), e0154128. https://doi.org/10.1371/journal.pone.0154128

[12] Maddock, R. J., \& Frein, S. T. (2009). Reduced memory for the spatial and temporal context of unpleasant words. Cognition and Emotion, 23(1), 96-

117. https://doi.org/10.1080/02699930801948977

[13] Rasch, B., \& Born, J. (2013). About Sleep's Role. in Memory. Physiological Reviews, 93(2), 681-766. https://doi.org/10.1152/physrev.00032.2012

[14] Peigneux, P., Orban, P., Balteau, E., Degueldre, C., Luxen, A., Laureys, S., \& Maquet, P. (2006). Offline Persistence of Memory-Related Cerebral Activity during Active Wakefulness. PLoS Biology, 4(4). https://doi.org/10.1371/journal.pbio.0040100

[15] Lo, J. C., Chong, P. L., Ganesan, S., Leong, R. L., \& Chee, M. W. (2016). Sleep deprivation increases formation of false memory. Journal of sleep research, 25(6), 673-682. https://doi.org/10.1111/jsr.12436

[16] Krause, A. J., Simon, E. B., Mander, B. A., Greer, S. M., Saletin, J. M., Goldstein-Piekarski, A. N., \& Walker, M. P. (2017). The sleep-deprived human brain. Nature reviews. Neuroscience, 18(7), 404418. https://doi.org/10.1038/nrn.2017.55

[17] Pilcher, J. J., \& Walters, A. S. (1997). How Sleep Deprivation Affects Psychological Variables Related to College Students' Cognitive Performance.
Journal of American College Health, 46(3), 121126. https://doi.org/10.1080/07448489709595597

[18] Herrnstein, M., Coloma Andrews, L., \& Hausmann, M. (2014). Gender-stereotyping and cognitive sex differences in mixed- and same-sex groups. Archives of sexual behavior, 43(8), 16631673. https://doi.org/10.1007/s10508-014-0311-5

[19] Marr D. (1971). Simple memory: a theory for. archicortex. Philosophical transactions of the Royal Society of London. Series B, Biological sciences, 262(841), 23-81. https://doi.org/10.1098/rstb.1971.0078 